
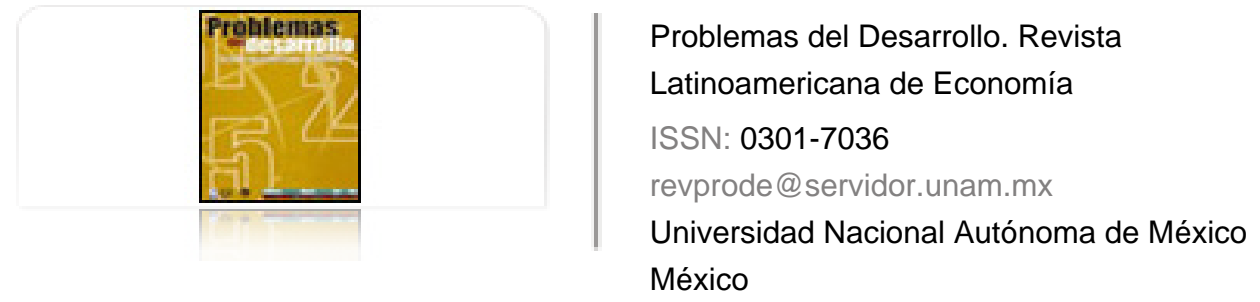

Palazuelos, Enrique

India en los albores de la transición energética

Problemas del Desarrollo. Revista Latinoamericana de Economía, vol. 39, núm. 154, julio-septiembre, 2008, pp. 11-36

Universidad Nacional Autónoma de México

Distrito Federal, México

Disponible en: http://www.redalyc.org/articulo.oa?id=11820139002

Cómo citar el artículo

- Número completo

- Más información del artículo

Página de la revista en redalyc.org

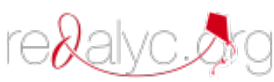

Sistema de Información Científica

Red de Revistas Científicas de América Latina, el Caribe, España y Portugal Proyecto académico sin fines de lucro, desarrollado bajo la iniciativa de acceso abierto 


\title{
INDIA EN LOS ALBORES DE LA TRANSICIÓN ENERGÉTICA
}

\author{
Enrique Palazuelos*
}

Fecha de recepción: 24 de enero de 2008. Fecha de aceptación: 8 de abril de 2008.

\section{Resumen}

Desde mediados de la última década del siglo $\mathrm{XX}$, el sistema energético de India viene registrando síntomas novedosos que marcan el comienzo de una transición en la que el funcionamiento de la oferta y la demanda de energía tenderá a corresponderse con los requisitos del desarrollo económico. En primer lugar, el trabajo examina los cambios energéticos recientes y después analiza su relación con el proceso de transformación económica y social que vive el país, con el fin de conocer cuáles son los factores que están determinando el inicio de la transición energética. Después muestra cómo las tendencias que se derivan de esos factores económicos y sociales inducen al aumento y la diversificación creciente de la demanda energética. Desde esa perspectiva y conforme a la dotación disponible de recursos energéticos, se analiza por qué la profundización de la transición energética conducirá, inexorablemente, a una mayor dependencia externa, sobre todo en petróleo, pero también en gas natural y en uranio.

Palabras clave: transición energética, India, desarrollo económico, sistema energético, dependencia energética.

* Catedrático de economía aplicada por la Universidad Complutense de Madrid. Correo electrónico:epalazue@ccee.ucm.es.

Este trabajo forma parte del proyecto del Grupo de Investigación sobre Crecimiento de la Economía Mundial (GICEM) Análisis comparativo de los sistemas energéticos de las tres grandes regiones de la economía mundial (Unión Europea, América del Norte y Asia Oriental) que son altamente dependientes en energía. El proyecto cuenta con el financiamiento del gobierno de la Comunidad Autónoma de Madrid por medio del Programa de Creación y Consolidación de Grupos de Investigación UCM-CM en el marco del IV Plan Regional de Investigación. En la elaboración de este trabajo he contado con la colaboración de Raquel del Amo Álvarez. 
Abstract

Since the middle of the 1990s, India's energy system has been registering new symptoms marking the commencement of a transition in which the functioning of energy demand and supply will have to correspond to the requirements of economic development. In the first place, this paper examines the recent energy changes, after which it analyzes their relationship with the process of economic and social transformation the country is experiencing, with the aim of discovering the factors that are determining the beginning of the energy transition. It then shows how the trends derived from those economic and social factors are inducing the increase and growing diversification in energy demand. From that perspective -and in line with the available supplies of energy resources -we analyze why the deepening of the energy transition will lead inexorably to greater external dependence, above all for petroleum, but also for natural gas and uranium.

Key words: energy transition, India, economic development, energy system, energy dependence.

\section{Résumé}

Depuis la seconde moitié du XXe siècle, le système énergétique de l'Inde enregistre des symptômes inconnus jusqu'alors qui marquent le début d'une étape de transition au cours de laquelle le fonctionnement de l'offre et de la demande tendra à répondre aux exigences $d u$ développement économique. Dans une première phase du travail sont examinés les changements récents dans le domaine de l'énergie, puis analysée leur relation avec le processus de transformation économique et sociale que vit le pays, dans le but de savoir quels sont les facteurs qui déterminent le démarrage de la transition énergétique. Ensuite, il est montré comment les tendances qui dérivent de ces facteurs économiques et sociaux induisent la hausse et la divesification croissante de la demande d'énergie. Une analyse selon cette perspective et considérant les ressources énergétiques disponibles explique pourquoi l'approfondissement de la transition énergétique conduira inexorablement à une plus grande dépendance de l'extérieur, surtout en ce qui concerne le pétrole mais aussi pour le gaz, naturel et l'uranium.

Mots clés: transition énergétique, Inde, développement économique, système énergétique, dépendance énergétique.

\section{Resumo}

Desde meados da última década do século XX o sistema energético da Índia vem registrando novos sintomas que marcam o começo de uma transição na que o funcionamento da oferta e a demanda de energia tenderão a corresponder com os requisitos do desenvolvimento econômico. Em primeiro lugar, o trabalho examina as mudanças energéticas recentes e depois analisa sua relação com o processo de transformação econômica e social que vive o país, com o fim de conhecer quais são os fatores que estão determinando o inicio da transição energética. Depois mostra como as tendências que se derivam desses fatores econômicos e sociais induzem ao aumento e a diversificação crescente da demanda energética. Desde esta perspectiva e conforme a dotação disponível de recursos energéticos se analisa por que a profundidade da transição energética conduzirá, inexoravelmente, a uma maior dependência externa, sobre todo no petróleo, mas também no gás natural e no urânio.

Palavras chave: transição energética, Índia, desenvolvimento econômico, sistema energético, dependência energética. 
Introducción

D esde mediados de la última década del siglo XX están surgiendo en India los primeros síntomas de cambio energético, influidos por las nuevas condiciones económicas creadas a partir de las reformas de 1991. El signo más evidente de ese cambio es la emergencia de India como un gran importador de petróleo, al mismo tiempo que lo hace China, el otro gigante asiático. Esta coincidencia, unida al colosal tamaño demográfico de ambos países y al dinamismo de sus economías, ha dado lugar a que muchos estudios presenten la situación energética de India y China como si fueran similares. Sin embargo, la insistencia en esas similitudes induce a equívocos importantes, pues los indicadores ponen de manifiesto grandes diferencias entre las dos naciones asiáticas y señalan que India ocupa posiciones bastante más rezagadas, tanto en términos de desarrollo económico como de cambio energético. De hecho, el análisis del sistema energético se convierte en una radiografía reveladora del profundo subdesarrollo que aflige a India y pone de manifiesto que todavía se encuentra en los prolegómenos de la transición hacia un funcionamiento energético más avanzado.

Las dos hipótesis centrales que sustentan este trabajo son las siguientes: en primer lugar, el proceso de transformación económica y social que vive India está determinando el surgimiento de unos cambios energéticos incipientes, que tendrán que cobrar mayor envergadura para que la transición energética se profundice. En segundo lugar, debido a la debilidad de recursos fósiles, esa profundización conducirá, inexorablemente, a una mayor dependencia frente al exterior. En consecuencia, el trabajo se ocupa de analizar los cambios energéticos recientes y los factores que los determinan, para después argumentar cuáles son las principales tendencias de la situación actual y por qué seguirá aumentando la dependencia exterior.

El artículo está organizado en cuatro apartados. El primero examina los rasgos que caracterizan el funcionamiento de la oferta y demanda de energía de India y su correspondencia con los cánones tradicionales de comportamiento energético de los países con bajo nivel de desarrollo. El segundo apartado aborda los cambios recientes y los rasgos de la dinámica económica y social que están determinando el inicio de la transición. El tercero analiza las tendencias que inducen al aumento y a la diversificación creciente de la demanda, al razonar por qué esas tendencias darán lugar a un desajuste cada vez mayor con respecto de la oferta de petróleo y, en menor medida, de gas natural, lo que ocasiona una mayor dependencia de ambos recursos. El cuarto apartado expone las conclusiones del trabajo.

Vol. 39, núm. 154, julio-septiembre / 2008

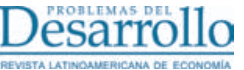




\section{El sistema energético característico de una economía subdesarrollada}

Las economías con un bajo nivel de desarrollo se caracterizan por disponer de una débil capacidad de acumulación, una estructura productiva asentada en actividades primarias y otras que aportan una escasa transformación, así como una distribución de la renta que reproduce ese tipo de especialización productiva y esa precaria acumulación. De ese modo, la industria y los servicios están poco desarrollados, las infraestructuras son escasas, la mayoría de la población vive en el medio rural, el ingreso medio per capita es reducido y los indicadores de bienestar social señalan grandes carencias para la mayoría de los habitantes. En consonancia con este atraso estructural, el sistema energético se caracteriza por un consumo final de energía de escasa magnitud y poco dinamismo, concentrado en el sector residencial -mayoritariamente rural—, donde cubre las actividades domésticas más básicas (cocina, calentamiento de agua y del hábitat); para ello emplea recursos energéticos primarios a los que se tiene acceso con medios técnicos poco mecanizados, y ocasiona un grave impacto ambiental sobre las superficies boscosas. Los sectores productivos tienen una participación menor en el consumo final; la oferta de recursos está poco diversificada y se corresponde con la escasa variedad que presenta la demanda, por ello existe un equilibrio bastante estable entre oferta y demanda, y apenas requiere la participación en el comercio internacional de productos energéticos. Estos rasgos se alteran sólo en aquellos países con reservas importantes de algún recurso energético, lo que les permite disponer de una oferta excedentaria que les convierte en exportadores de dicho recurso.

Con los matices y las novedades que se analizan más adelante, el sistema energético de India muestra una acusada similitud con las características que se acaban de exponer, de manera que el patrón básico de oferta-demanda de energía responde a los rasgos de un país de bajo desarrollo. El análisis de los balances energéticos permite concretar los rasgos principales de ese sistema energético.

a) Modesto nivel y escaso dinamismo del consumo final de energía (CFE). En 2004, el consumo apenas superaba los 400 millones de toneladas equivalentes de petróleo (tep) $)^{1}$ y pese a que el Producto Interno Bruto (PIB) registró un incremento de

1 China, el otro gigante asiático con el que frecuentemente se compara la evolución hindú, tenía en 2004 un CFE de 1,038 millones de tep, esto es 2.6 veces al de India. Fuente: International Energy Agency (2006a).

\section{DeSTarrollo}


110\% entre 1991 y 2004, el CFE sólo creció 37\%, lo que responde con debilidad al dinamismo de la economía.

b) Concentración del consumo de energía en el sector residencial. A pesar del lento aumento del consumo, su participación se mantiene por encima de 57\% del CFE (cuadro 1). A distancia, la industria representa $30 \%$, debido sobre todo a la alta participación de la rama de química y petroquímica que absorbe más de la quinta parte del consumo industrial, y el resto se reparte de forma dispersa entre las demás ramas industriales, incluyendo la minería y la construcción. En consecuencia, los demás sectores apenas tienen relieve en el consumo final, ya que el transporte significa menos de $9 \%$ y tanto el sector comercial (que incluye al conjunto de servicios privados y públicos) como el sector primario representan porcentajes de $1.5 \%$.

c) La fuerte concentración sectorial respecto al sector residencial se corresponde, desde el punto de vista de los recursos consumidos, con el abrumador predominio de los combustibles sólidos renovables, es decir, madera, estiércol y otras biomasas compuestas de desechos vegetales y animales, que suponen más de la mitad del CFE. A gran distancia, el segundo recurso utilizado son los derivados de petróleo, cuyo rápido crecimiento ha elevado su participación hasta superar $25 \%$. De ese modo, las restantes fuentes sólo cubren otra cuarta parte del CFE, aunque con distintas trayectorias: el carbón tiene cada vez menos presencia, mientras la electricidad y el gas natural avanzan con rapidez pero aún registran cuotas modestas.

Cuadro 1

PIB, demanda total y consumo final distribución por sectores, 1991-2004

\begin{tabular}{|c|c|c|c|c|c|c|}
\hline & \multicolumn{3}{|c|}{ Tasas medias de crecimiento anual (\%) } & \multicolumn{3}{|c|}{ Participación en el consumo final de energía (\%) } \\
\hline & 1991-1995 & $1996-2000$ & $2007-2004$ & 1990 & 2000 & 2004 \\
\hline PIB & 5.2 & 5.8 & 6.2 & & & \\
\hline Demanda total de energía primaria & 3.8 & 3.2 & 2.8 & & & \\
\hline Consumo final de energía & 2.6 & 2.1 & 2.1 & 100 & 100 & 100 \\
\hline Sector residencial & 1.8 & 1.8 & 2.0 & 59.4 & 57.9 & 57.5 \\
\hline Sector industrial & 2.5 & 4.4 & 2.6 & 26.5 & 29.6 & 30.2 \\
\hline - Usos energéticos & 1.8 & 3.0 & 1.3 & 24.2 & 24.4 & 23.7 \\
\hline - Usos no energéticos & 9.1 & 13.4 & 7.7 & 2.3 & 5.2 & 6.5 \\
\hline * Química-petroquímica & 6.2 & 5.7 & 0.8 & 4.8 & 6.8 & 6.5 \\
\hline Sector transporte & 5.6 & -2.1 & 2.0 & 10.2 & 8.9 & 8.9 \\
\hline * Carretera & 8.4 & -2.6 & 1.7 & 8.5 & 7.5 & 7.5 \\
\hline Sector comercial y servicios & 2.9 & -2.1 & 2.9 & 1.6 & 1.5 & 1.5 \\
\hline Sector primario & 11.1 & -0.1 & 1.1 & 2.1 & 2.1 & 2.0 \\
\hline
\end{tabular}

Fuente: Elaboración a partir de datos de la International Energy Agency (IEA) (2006a).

Vol. 39, núm. 154, julio-septiembre / 2008 
d) La transformación de recursos primarios sigue siendo limitada, aunque se ha intensificado desde los años noventa como resultado de la creciente participación de los derivados de petróleo y de la electricidad en el CFE. Por un lado, entre 1991 y 2004, la generación de energía eléctrica ha crecido a una tasa media superior a 6\% anual, hasta 668 Teravatios-hora (TWh). La contribución de las plantas térmicas se ha incrementado de $78 \%$ a $85 \%$ de esa producción eléctrica, merced a que se mantiene la mayoritaria aportación del carbón (70\%) y elevan la suya las centrales que utilizan gas natural $(9 \%)$ y derivados de petróleo (6\%). Por consiguiente, las plantas que funcionan con combustibles no fósiles tienen una participación minoritaria y declinante, ya que la aportación de la energía nuclear avanza con lentitud (3\%) y la energía hidráulica reduce la suya (12\%), mientras que las otras energías renovables ocupan un lugar testimonial.

Por otro lado, la producción de derivados de petróleo también ha registrado un fuerte crecimiento a partir de la segunda mitad de los años noventa. Entre 1995 y 2004, el procesamiento de petróleo crudo pasó de 60 a 132 millones de toneladas, lo que supone un ritmo de crecimiento de $13 \%$ anual. Se incrementa así la disponibilidad de productos destinados a la generación de electricidad (combustóleo y diesel), la industria (diesel, naftas), el transporte (diesel, gasolina) y el consumo doméstico (keroseno y componentes ligeros).

e) La demanda total de energía primaria (DTEP) se ha visto más estimulada por el consumo intermedio que por los sectores finales. A pesar de ello, su tasa media de crecimiento (3\% anual) es casi la mitad de la registrada por el PIB (5.8\%) en el intervalo 1991-2004 y el nivel que alcanza en el último año (573 millones de tep) sigue siendo modesto. Por ejemplo, apenas equivale a la tercera parte de la DTEP de China, cifrada en 1,600 millones de tep. La composición de la demanda refleja una presencia del carbón (33\%) muy superior a la que tiene en el CFE, debido a su gran participación en la generación de electricidad. Sin embargo, el principal recurso sigue siendo el empleo de combustibles sólidos renovables que todavía mantienen una cuota cercana a $40 \%$ de la DTEP (cuadro 3).

f) La intensidad energética, medida por la relación DTEP/PIB, tiende a reducirse, pero sigue arrojando valores elevados. Desde que a finales de los años setenta alcanzó su máximo, cercano a 0.30 tep por mil dólares [constantes de 2000 en PPA (paridad del poder adquisitivo)], el valor fue bajando hasta situarse en 0.18 tep por mil dólares en 2004. En esa trayectoria descendente influyen factores de distinto orden, entre los que están la mejora de la eficiencia técnica conseguida en el uso de los recursos energéticos, la modificación de la estructura sectorial de la 
producción y la sustitución de unas fuentes energéticas de menor calidad por otras con mayores ventajas caloríficas o lumínicas o para otros usos (Schipper y Grubb, 2000; Judson et al., 1998). Sin embargo, su nivel actual sigue siendo muy alto pues se trata de una intensidad similar a la que presenta el promedio de la Unión Europea (UE), es decir, economías con capacidades industriales, sector de transporte, servicios y bienestar social muy superiores a los de India. Esa intensidad energética expresa la permanencia de importantes dosis de ineficiencia, además de una débil especialización productiva y una insatisfactoria composición de la demanda de energía (ECEP, 2005). ${ }^{2}$

\section{Gráfica 1}

Índices de crecimiento del PIB, la población y la demanda de energía $(1980=100)$. Intensidades energéticas respecto al PIB y a la población. PIB (en la ordenada derecha), 1980-2004

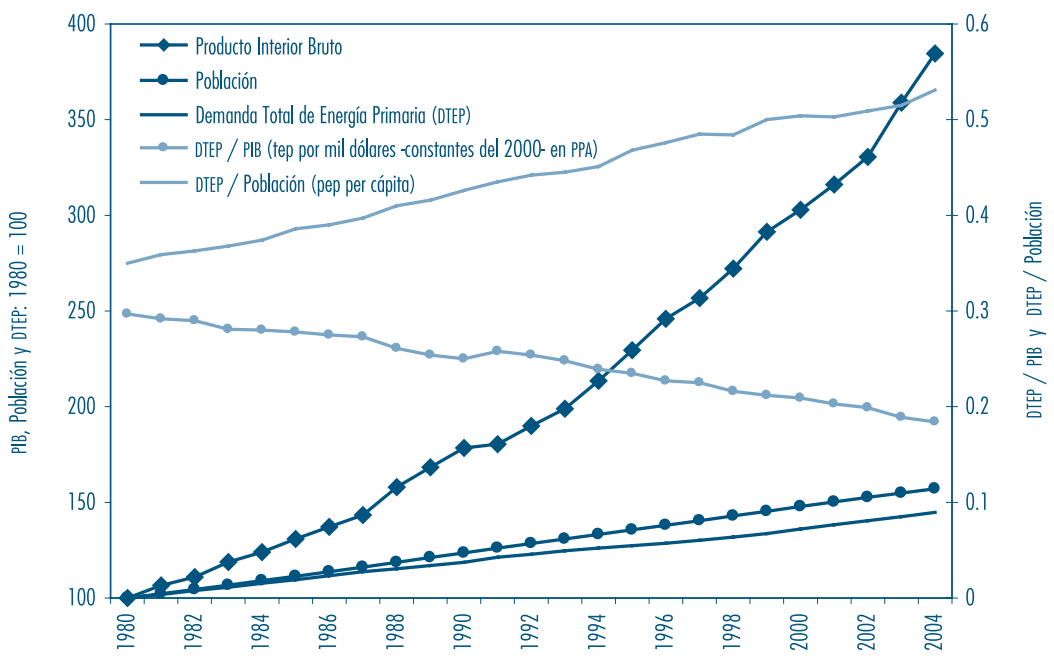

Fuente: Elaborado a partir de IEA (2006a).

2 El bajo rendimiento de los recursos energéticos se aprecia de forma aún más elocuente en la intensidad del consumo de electricidad, cuyo crecimiento se prolongó hasta mediados de los noventa hasta un máximo de $0.98 \mathrm{kWh}$ por dólar de PIB y el descenso posterior ha situado la relación en $0.85 \mathrm{kWh} / \$$. Este valor es bastante más alto que el que muestran países de desarrollo medio, como Portugal o Turquía $(0.50-0.40 \mathrm{kWh} / \$)$, y es exageradamente mayor que el promedio de la Unión Europea (0.31), pese a que a ésta pertenecen países cuyo grado de electrificación es muy superior al de India, tanto en la industria y los servicios como en el alumbrado público y el consumo de los hogares. Fuente: IEA (2006a).

Vol. 39, núm. 154, julio-septiembre / 2008

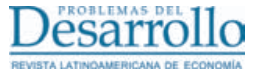


g) El indicador que pone definitivamente al descubierto la precariedad energética de India es el que relaciona la DTEP con el volumen de población. Aunque ha ido aumentando en el transcurso de las últimas décadas, su valor en 2004 es de sólo 0.531 tep por habitante. Basta recordar que la intensidad energética per capita de China es 2.5 veces mayor, la de Portugal lo es por cinco y el promedio de la Unión Europea por ocho. En el caso del consumo de electricidad per capita, el nivel actual de India ( $457 \mathrm{KWh}$ per capita) es 3.5 veces inferior al de China, casi 10 veces menor que el de Portugal y 15 veces menor que la media de la UE.

h) La correspondencia entre oferta y demanda de energía reposa fundamentalmente en la disponibilidad de madera y biomasas orgánicas con los que se abastece el consumo de un sector residencial compuesto en más de $70 \%$ por hogares rurales. En un segundo rango, el carbón, requerido sobre todo para generar energía eléctrica, también se cubre mayoritariamente con producción nacional. ${ }^{3}$ Con menor incidencia, pues su presencia en la DTEP es reducida, lo mismo sucede con el gas natural ${ }^{4}$ que se abastece con producción interna. El único desajuste se presenta en la utilización de petróleo, pues la producción de petróleo crudo es reducida y apenas crece, mientras que la demanda de derivados sí lo hace con celeridad por las razones que se analizan más adelante.

Cuadro 2

Distribución del consumo final por fuentes, 1991-2004

\begin{tabular}{|c|c|c|c|c|}
\hline & \multicolumn{3}{|c|}{ Participación en el consumo final de energía (\%) } & \multirow{2}{*}{$\frac{\text { Tasa media de crecimiento anual }(\%,}{1997-2004}$} \\
\hline & 1991-1995 & $1996-2000$ & $2001-2004$ & \\
\hline Combustibles sólidos renovables & 58.0 & 54.9 & 53.8 & 1.4 \\
\hline Derivados de petróleo & 19.7 & 24.1 & 25.9 & 5.0 \\
\hline Carbón & 12.7 & 10.0 & 8.9 & -0.9 \\
\hline Electricidad & 7.3 & 8.4 & 9.1 & 5.5 \\
\hline Gas natural & 2.3 & 2.6 & 2.3 & 3.0 \\
\hline
\end{tabular}

Fuente: Elaboración a partir de datos de la IEA (2006a).

3 Con el paso del tiempo, la proporción de carbón destinado a las plantas térmicas y a usos finales (industrias de siderúrgica, cemento y otras) se ha modificando desde una relación 53-47\% en 1990 a otra de 75-25\% en 2004. Fuente: Koizumi (2006), citando datos de la Central Statistical Organization.

4 Casi $60 \%$ de la demanda de gas procede de las plantas térmicas, $35 \%$ de la industria y el resto de los otros sectores finales. Fuente: Koizumi (2006), citando datos de la Central Statistical Organization.

\section{DeSTarrollo}

Vol. 39, núm. 154, julio-septiembre / 2008 
Cuadro 3

Evolución y distribución de la demanda y de la producción de energía primaria

\begin{tabular}{|c|c|c|c|c|c|}
\hline & \multicolumn{3}{|c|}{ Tasas medias de crecimiento anual (\%) } & \multicolumn{2}{|c|}{ Promedio 2001-2004 } \\
\hline & $1997-1995$ & $1996-2000$ & $2001-2004$ & Millones de tep & $\%$ \\
\hline Demanda total & 3.8 & 3.2 & 2.8 & 543.8 & 100 \\
\hline Combustibles sólidos renovables & 1.4 & 1.3 & 1.6 & 209.6 & 38.6 \\
\hline Carbón & 5.5 & 3.5 & 4.5 & 178.9 & 32.9 \\
\hline Petróleo crudo & 3.6 & 11.1 & 5.0 & 126.9 & 23.3 \\
\hline Gas natural & 12.0 & 3.9 & 22.8 & 22.5 & 4.1 \\
\hline Hidráulica & 0.3 & 0.5 & 3.3 & 6.4 & 1.2 \\
\hline Nuclear & 5.4 & 16.2 & 0.2 & 4.8 & 0.9 \\
\hline Eólica y solar & 71.9 & 26.4 & 22.2 & 0.3 & 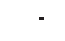 \\
\hline Producción & 2.9 & 1.6 & 2.9 & 445.0 & 100 \\
\hline Combustibles sólidos renovables & 1.4 & 1.3 & 1.6 & 209.6 & 47.1 \\
\hline Carbón & 4.6 & 2.3 & 5.0 & 163.2 & 36.7 \\
\hline Petróleo crudo & 2.1 & -1.0 & 1.3 & 38.2 & 8.6 \\
\hline Gas natural & 12.0 & 3.9 & 22.8 & 22.5 & 5.1 \\
\hline Hidráulica & 0.3 & 0.5 & 3.3 & 6.4 & 1.4 \\
\hline Nuclear & 5.4 & 16.2 & 0.2 & 4.8 & 1.1 \\
\hline Eólica y solar & 71.9 & 26.4 & 22.2 & 0.3 & -0.1 \\
\hline
\end{tabular}

Fuente: Elaboración a partir de datos de la IEA (2006a).

\section{Los primeros cambios energéticos y sus determinantes}

Al observar con detalle lo que ha sucedido desde 1991 se perciben varias novedades que han comenzado a modificar las bases del sistema energético. Los cambios apenas se captan desde la perspectiva sectorial, pues casi la mitad del moderado crecimiento que ha registrado el consumo final (en 110 millones de tep) entre 1991 y 2004 corresponde al consumo residencial y otro $40 \%$ se debe al sector industrial, de manera que a los demás sectores sólo les corresponde $12 \%$ del incremento del CFE. Sin embargo, desde la perspectiva de las fuentes que abastecen a esos sectores sí se aprecian modificaciones relevantes. Casi la mitad del incremento del CFE ha sido de derivados de petróleo, $20 \%$ de electricidad y, con modestia, $4 \%$ de gas natural, de manera que esos tres recursos de mayor calidad energética han comenzado a sustituir al consumo de biomasas y de carbón, ${ }^{5}$ tanto en la industria como en el sector residencial.

5 Los combustibles sólidos renovables han sido $34 \%$ del aumento del CFE, mientras que el consumo de carbón ha retrocedido en 4.4 millones de tep, es decir, $-4 \%$ del aumento del CFE.

Vol. 39, núm. 154, julio-septiembre / 2008

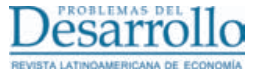


A su vez, la mayor demanda de derivados y de electricidad es la que exige el rápido aumento de la capacidad eléctrica y de procesamiento de petróleo crudo, aunque con una diferencia fundamental. Mientras que la producción eléctrica se surte de recursos internos (carbón y gas natural), las refinerías aumentan su producción con petróleo crudo importado. Al mismo tiempo, esa mayor capacidad de procesamiento ha dado lugar a un cambio de signo en el comercio de productos petrolíferos, de los que India se ha convertido en exportadora neta. Se trata, pues, de dos tipos de desajuste ofertademanda de sentido contrario. Ambos promueven una mayor inserción del sistema energético hindú en el comercio internacional, si bien el flujo de mayor envergadura es el que tiene lugar mediante la importación de petróleo crudo, que se amplía con el paso del tiempo y conlleva un grado creciente de dependencia externa. En 2004 la importación de petróleo crudo equivalía a 79\% de la demanda interna de petróleo, cifrada en 120.7 millones de toneladas (gráfica 2), haciendo que la presencia de India en el mercado internacional de petróleo crudo se eleve desde $1.6 \%$ que ostentaba a mediados de los años noventa hasta $4.4 \%{ }^{6}$

Gráfica 2

Importaciones y exportaciones de petróleo, 1980-2004

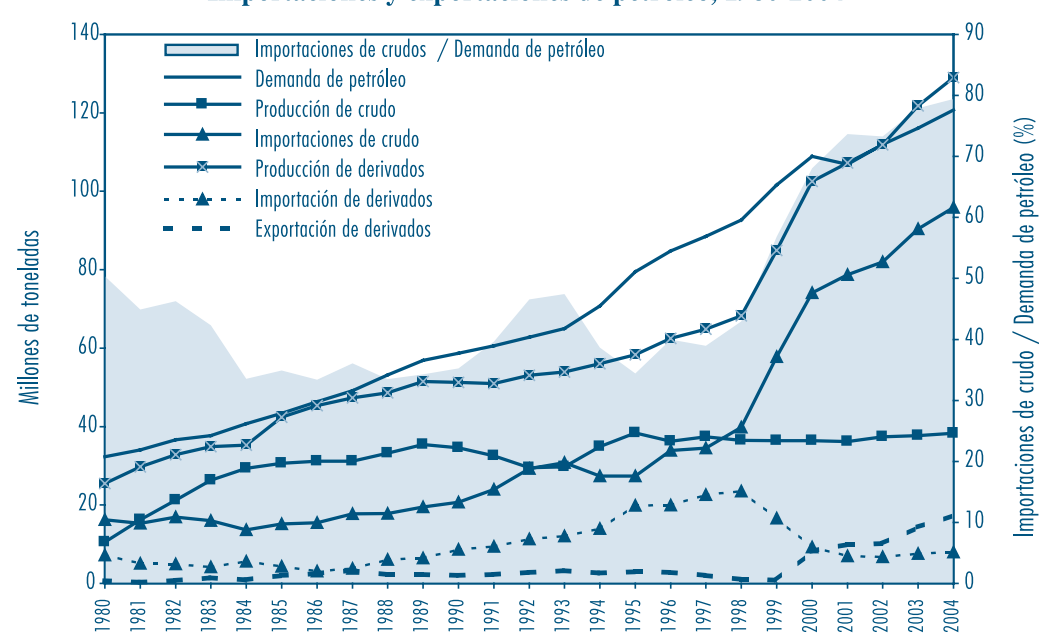

Fuente: Elaborado a partir de IEA (2006b).

6 El dato avanzado para 2005 por la IEA (2006b) confirma que las importaciones continúan su crecimiento de forma acelerada, al pasar de 98 a 121 millones de tep, elevando su cuota en las compras mundiales hasta 5.3\%. En el caso de los productos refinados, durante la primera mitad de los noventa las compras crecieron de 9 a 20 millones de tep y las exportaciones se mantuvieron en 2-3 millones; pero en la segunda mitad de la década, tras la rápida expansión de las

\section{DeSTarrollo}


Alcance de las modificaciones económicas y sociales

La nueva política económica aplicada desde 1991 se propuso lograr el ajuste externo y el reequilibrio de las cuentas públicas mediante la aplicación de medidas contractivas de demanda y de rentas, dirigidas a la contención del gasto agregado, a la vez que introdujo sucesivas medidas liberalizadoras para reducir el control del Estado sobre la economía, así como estimular al sector privado, ampliar el comercio exterior y atraer inversiones extranjeras (Bajpai 1999; Chai y Roy, 2006). Tras un primer momento de impasse, mientras reajustaba las variables fiscales y externas y adaptaba su funcionamiento a las medidas liberalizadoras y de apertura externa, la economía recuperó a partir de 1993 una dinámica de rápido crecimiento: la tasa media del intervalo 1991-2004 casi alcanzó $6 \%$ anual, acompañada de importantes modificaciones estructurales.

Desde la perspectiva de la demanda, la inversión fija se ha fortalecido mediante un crecimiento (7.2\%) más intenso que el obtenido por el consumo privado (4.7\%) y público (4.9\%). Pero más decisivo aún ha sido el comportamiento de sus componentes externos, ya que las exportaciones e importaciones de bienes y servicios han alcanzado tasas de crecimiento de $13.1 \%$ y $12.2 \%$, respectivamente, las cuales han elevado con rapidez la relación de las exportaciones (de 7\% a 18.1\%) y las importaciones (de $6.9 \%$ a $15.8 \%$ ) respecto al PIB. Significativamente, el saldo externo positivo que reflejan esos datos no procede del intercambio de bienes, sino del excelente registro de la balanza de servicios cuyo superávit equivale a 5\% del PIB (ADB, 2007).

Desde la perspectiva de la oferta, se observa una fuerte alteración de la estructura sectorial de la producción. La agricultura, con una tasa media de $2.5 \%$ anual, sólo ha respondido de forma parcial al dinamismo de la economía. El sector industrial sí se ha activado más, pero los mayores crecimientos han correspondido al sector terciario, debido al efecto conjunto de las medidas liberalizadoras en la expansión del comercio, las finanzas y otras actividades, y del auge adquirido por los denominados ITES-BPO (Information Technology -Services and Business Process Outsourcing) al calor de la apertura externa y la entrada de inversión extranjera. Respecto a un fuerte sector de software informático, integrado por grandes corporaciones transnacionales y compañías nacionales, se ha formado una red de servicios instrumentales para ese sector y se han asentado numerosos negocios de subcontratación vinculados a empresas internacionales que deslocalizan esos servicios (administrativos, contabilidad,

refinerías, las importaciones retrocedieron y las exportaciones aumentaron (gráfica 1), al arrojar un saldo neto exportador cercano a los 10 millones de tep. En el caso del carbón, se importan cantidades cercanas a 9\% de su demanda interna, y se concentran en la adquisición de coque.

Vol. 39, núm. 154, julio-septiembre / 2008 
call centres y otros) de los países desarrollados a los que pertenecen. La abundancia de mano de obra muy barata que domina el idioma inglés favorece la apertura de esos negocios subcontratados (EIU, 2007; Chauvin-Lemoine, 2005).

En apenas década y media la estructura del PIB ha experimentado una fuerte convulsión (cuadro 4). La agricultura ha perdido casi 12 puntos, ganados por los servicios, en particular comercio, transporte y comunicaciones (siete puntos) y finanzas (tres), mientras que el sector industrial sólo ha elevado un punto su contribución gracias a la construcción. Resulta sintomático de las características actuales de la economía hindú el hecho de que —en un contexto de fuerte crecimiento de la economía— las manufacturas no hayan modificado su (débil) aportación, que se mantiene en torno a $16 \%$ del PIB.

Desde la perspectiva institucional, la economía también ha experimentado notables alteraciones. Las medidas liberalizadoras han favorecido la creación de empresas privadas, sobre todo en los servicios, y la entrada de compañías extranjeras en actividades que hasta entonces sólo contaban con empresas estatales. En el mismo sentido, se han ido relajando las regulaciones que pesaban sobre la casi totalidad de los precios y sobre las entidades financieras, se han reestructurado las empresas estatales, se han reducido los subsidios públicos y ha disminuido el proteccionismo comercial. Simultáneamente, en la estructura social, el crecimiento del ingreso y la menor presión demográfica otorgan una mayor capacidad de consumo a ciertos núcleos de la población, relacionados sobre todo con el aumento de la población que vive en las ciudades, además del paulatino aumento de la educación y la capacidad técnica de nuevos segmentos de la población.

Cuadro 4

Estructura sectorial del PIB y crecimiento de los sectores: 1990-2004

\begin{tabular}{lcrcc}
\hline & 1990 & 2004 & 2006 & Tasa de crecimiento 1990-2004 \\
\hline PIB & 100 & 100 & 100 & 5.8 \\
Agricultura & 29.3 & 18.7 & 17.5 & 2.5 \\
Minería & 2.7 & 3.0 & 2.7 & 4.3 \\
Manufactura & 16.7 & 15.9 & 16.2 & 5.8 \\
Electricidad, agua y gas & 2.1 & 2.1 & 2.0 & 6.2 \\
Construcción & 5.4 & 6.5 & 6.9 & 6.3 \\
Transporte, comunicaciones y comercio & 18.9 & 25.0 & 25.9 & 8.4 \\
Bancos, seguros y otras instituciones financieras & 11.6 & 14.5 & 14.4 & 7.7 \\
Administración y otros servicios & 13.3 & 14.3 & 14.3 & 6.1 \\
\hline
\end{tabular}

Fuente: Elaborado a partir de datos de ADB (2007), Key indicators. 
Se trata de modificaciones relevantes que, sin embargo, no deben inducir al equívoco de exagerar su alcance, puesto que los hechos muestran que la economía sigue sometida a los lastres que acarrea el subdesarrollo. El PIB per capita no alcanzaba los 3,000 dólares-PPA en 2004. La estructura económica acusa una pavorosa insuficiencia de capital. La agricultura crece con lentitud, siendo el sector que alberga a $60 \%$ del empleo y proporciona el sustento básico de la mayoría de los hogares rurales. La industria manufacturera mantiene una especialización basada en producciones con escaso valor añadido, donde sólo de forma puntual destacan algunas líneas más avanzadas, vinculadas al sofware informático, la química de farmacia y la fabricación de juguetes y prendas deportivas en condiciones de outsourcing. Esa estrechez manufacturera se manifiesta también en el notable déficit que arroja el comercio exterior de bienes (Chauvin-Lemoine, 2005). La expansión de las actividades comerciales y financieras, influidas por las reformas, sigue mostrando que el grueso de las actividades está en manos de un gran número de pequeñas empresas que carecen del capital, el tamaño y la capacidad de gestión para alcanzar los niveles de eficiencia propios de las economías modernas. La dotación de infraestructuras físicas sigue padeciendo un lamentable atraso, en particular el ferrocarril, la red de carreteras y las telecomunicaciones. Según los datos del Banco Mundial y el PNUD, citados por Madan (2006), $35 \%$ de los 1,100 millones de ciudadanos indios sigue teniendo ingresos inferiores a un dólar-PPA por día y 80\% de la población no alcanza los dos dólares-día. El 44\% de los hogares no tiene acceso a las redes de distribución de energía eléctrica, $16 \%$ carece de agua corriente y $47 \%$ de los niños menores de cinco años están malnutridos.

Dos hechos más resultan relevantes por la influencia que tienen en el comportamiento de las variables energéticas. Por un lado, la lentitud con la que avanza el proceso de urbanización, que desde 1990 sólo ha aumentado tres puntos hasta situarse en 29\% en 2004 (Madan, 2006). Por otro lado, la producción de automóviles sigue siendo muy pequeña (1.7 millones frente a los 5.2 millones de China) y el exiguo parque existente sólo equivale a 11 vehículos por cada mil habitantes, cuando en China alcanza el doble y la media de la Unión Europea es 35 veces superior (Perlitz, 2005).

\section{Determinantes de los cambios energéticos}

El análisis de la dinámica económica y social aporta los principales argumentos que explican los rasgos de continuidad del sistema energético tradicional y también los cambios que han sucedido en la última década y media. Si se comienza por los elementos de continuidad, el hecho de que el crecimiento económico esté sostenido por los servicios explica que el consumo energético presente una baja

Vol. 39, núm. 154, julio-septiembre / 2008

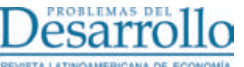


elasticidad-ingreso, pues las actividades terciarias que se han mostrado más dinámicas son escasamente intensivas en energía, lo mismo que las manufacturas tradicionales que más han crecido, las cuales se hallan pobremente mecanizadas. El menor crecimiento de las industrias de bienes de equipo junto al precario estado del transporte y otras infraestructuras hacen que tampoco aumenten con rapidez las producciones intermedias que son más intensivas en energía, como la siderurgia, el cemento, la metalurgia no ferrosa y la química industrial. El escaso parque de vehículos limita drásticamente el consumo de derivados de petróleos utilizados como combustibles.

Sin embargo, los aspectos más decisivos son los que conciernen al consumo residencial. El lento aumento de la población urbana y la enorme desigualdad de la distribución de la renta son los principales factores que explican el rígido comportamiento del consumo energético de los hogares, que crece con lentitud y apenas modifica su composición por fuentes. La aplastante mayoría de los hogares rurales sigue utilizando masivamente la leña, el estiércol y otros desechos para cubrir sus necesidades básicas. Los datos de Central Statical Organization (CSO) muestran que incluso en los hogares rurales de rentas medias y altas las biomasas constituyen el principal recurso energético, aunque sea en ellos donde se refleja una mayor presencia de derivados de petróleo y electricidad; conforme a las necesidades básicas añaden otras de refrigeración, disponibilidad de agua mediante bombeo e iluminación (gráfica 3).

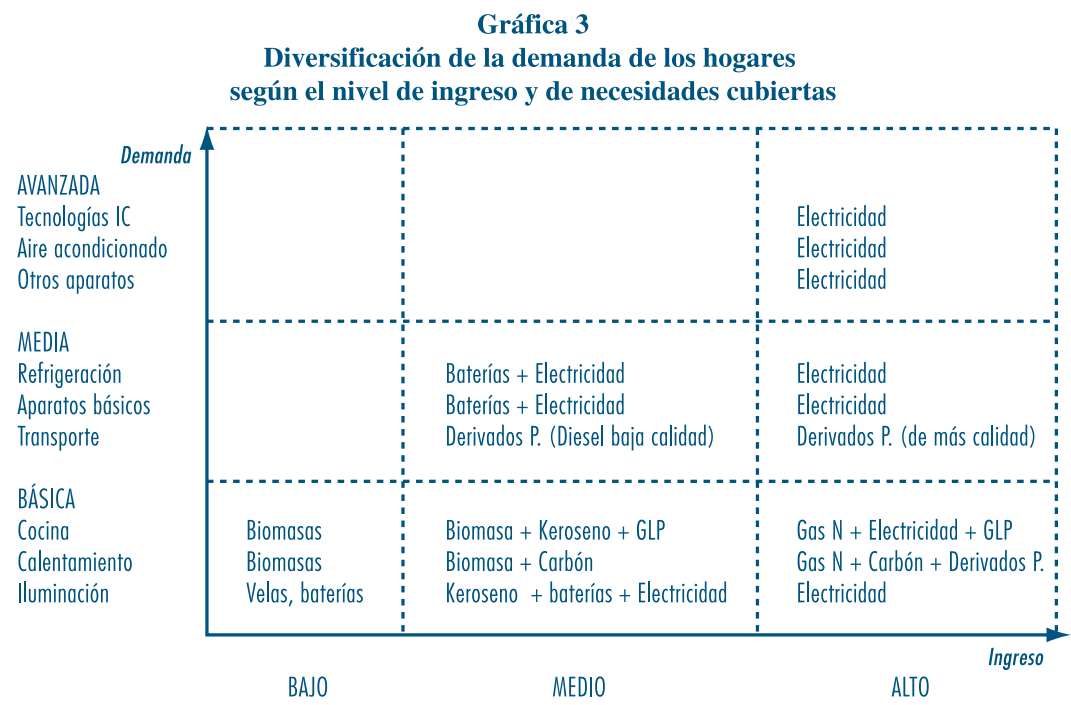

Fuente: Adaptado de la gráfica de IEA (2002: p. 370), World Energy Outlook. 
Los hogares urbanos son los que registran más cambios, aunque de forma pausada. Su ingreso medio supera varias veces el que tienen los hogares, de modo que es mayor el porcentaje de ciudadanos con acceso a aparatos electrodomésticos y a una cobertura más amplia de las necesidades básicas. En esa medida, esos hogares presentan una demanda más diversificada, donde las biomasas representan la quinta parte del consumo, elevando su contribución los componentes ligeros de petróleo, el keroseno, otros derivados y la energía eléctrica (Madan, 2006). No obstante, a pesar de esos cambios, sólo 16\% del consumo de los hogares indios se compone de recursos ajenos a las biomasas, repartiéndose entre $10 \%$ de productos petrolíferos, $3.5 \%$ de electricidad y $2.5 \%$ de carbón.?

Por tanto, al considerar el comportamiento de los sectores de consumo, es lógico que la intensidad energética haya descendido, pues, sea por sus características (servicios e industrias ligeras) o por su infradesarrollo (ramas de bienes de equipo e intermedias, transporte, hogares urbanos), ninguno de los sectores finales presenta una elasticidad significativa con respecto al crecimiento del PIB. Y, a la vez, los niveles que registra la intensidad energética siguen siendo altos porque las actividades productivas y domésticas que realizan ese consumo son bastante ineficientes y siguen utilizando recursos energéticos de baja calidad.

Pero, al mismo tiempo, son esas transformaciones socioeconómicas las que impulsan los incipientes cambios que se observan en el sistema energético: aumento de la demanda de electricidad y de productos refinados, desajuste entre la oferta y la demanda de petróleo, y rápido crecimiento de la dependencia energética.

El consumo de electricidad crece poco a poco en los sectores finales y, en consecuencia, también aumenta su producción, aunque la capacidad instalada sigue siendo modesta y la oferta no cubre de forma suficiente el incremento de la demanda. En

7 Hay que tener en cuenta que esos datos — calculados a partir de los balances energéticos de la IEA (2006a) — infravaloran el consumo de electricidad porque ignoran un fenómeno bastante generalizado en la periferia de las ciudades y en los pueblos de cierto tamaño. Las estadísticas registran que en la distribución de electricidad se producen grandes pérdidas, en una proporción que ha ido en aumento y en 2004 equivalía a más de la cuarta parte de la producción. Ciertamente, en parte, esas pérdidas se pueden imputarlas al mal estado de las redes que transportan el fluido eléctrico. Pero, al mismo tiempo, otra parte considerable de esas pérdidas son "desviaciones" que hacen los hogares de las zonas señaladas mediante enganches ilegales a las redes de distribución para obtener la electricidad que consumen, pero no queda computada como tal. La IEA ha examinado este hecho característico de los países subdesarrollados (World Energy Outlook, 2002) y de bolsas marginales que se agrupan en las periferias urbanas de muchos países desarrollados.

Vol. 39, núm. 154, julio-septiembre / 2008

Desarrollo 
2004 existía un desfase equivalente a 7\% de la demanda, que en las horas-pico, de máximo consumo, ascendía hasta 12\% (CSO, 2006). Sin embargo, los mayores desfases tienen lugar en el petróleo. El aumento de la demanda de derivados desde la industria, los hogares y el transporte ha exigido un fuerte crecimiento de la capacidad de procesamiento, pero no ha sido acompañado por un aumento de la producción de petróleo crudo. ${ }^{8}$ De ese modo, la demanda crece a costa de las importaciones y genera esa mayor dependencia exterior.

A la vista de los hechos analizados, la situación energética actual es ciertamente complicada puesto que se ha generado una elevada dependencia del petróleo importado sin que la economía haya fortalecido de forma significativa su "músculo productivo", es decir, su capacidad industrial. El aumento de la demanda de energía, y sobre todo el consumo de productos petrolíferos, está siendo determinado por unos factores que sólo impulsan modestamente el desarrollo económico y social.

\section{Transición energética y dependencia exterior}

Las autoridades indias y diversos organismos internacionales apuestan por un fuerte ritmo de crecimiento sostenido a largo plazo, que cifran en torno a $8 \%$ anual para los próximos 25 años, lo que supondría que el PIB se multiplicaría por siete y el PIB por capita aproximadamente por cinco. Pero esa perspectiva de crecimiento fuerte y sostenido no podrá alcanzarse sólo con los actuales resortes de crecimiento, sino que requiere el fortalecimiento de la estructura industrial y de las infraestructuras (transporte, suministros básicos, telecomunicaciones), mediante un proceso de especialización basado en actividades finales (de capital o consumo) con un mayor contenido tecnológico y en producciones intermedias (siderúrgicas, metalúrgicas, no metálicas, químicas, electricidad) que aporten los insumos transformados que necesitan las industrias finales y las infraestructuras. Implica también un grado de

8 Las refinerías mantienen una estructura de procesamiento orientada hacia el mercado interno, si bien generan cierto excedente que se exporta. En primer lugar, las refinerías producen diesel (35-40\%) para el transporte, la industria y el sector residencial. En segunda instancia, producen combustóleo (15-20\%) para las centrales térmicas y la industria química, y naftas (10-12\%) utilizadas como materia prima por esa misma industria. A continuación, producen gasolina (8-9\%), en proporciones menores debido a la modestia del parque de vehículos motorizados, mientras que el keroseno (8-9\%) tiene una participación cada vez menor porque su consumo en los hogares se sustituye por los componentes ligeros de petróleo (4-5\%) y por electricidad. El resto de la destilación de petróleo crudo se compone de keroseno para aviones y de productos residuales que se utilizan en la industria, o bien para el asfaltado de las calles y carreteras, y otros usos no energéticos.

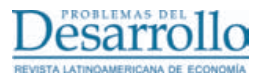

Vol. 39, núm. 154, julio-septiembre / 2008 
urbanización muy superior al actual, que las autoridades sitúan en torno a $45 \%$ en 2030 (Madan, 2006) con unas pautas de comportamiento social distintas de las tradicionales que continúan vigentes.

Adentrarse en esa perspectiva de desarrollo supondrá una conmoción de los cimientos en los que se asienta el sistema energético tradicional, para dar paso a un sistema moderno. En esa transición, la demanda de energía tendrá que adquirir una mayor elasticidad respecto al crecimiento del PIB, pues el fortalecimiento industrial y del transporte exigirá mayores consumos energéticos, de modo que - como estiman los cálculos oficiales - tenderá a incrementarse a una tasa media entre $4 \%$ y $5 \%$ anual (TERI, 2002; PCGI, 2002; ECEP, 2005), a la vez que irá diversificándose con una mayor presencia de los derivados de petróleo y la electricidad, pero también el carbón (para las plantas térmicas) y el gas natural, lo que supondrá una importante reducción del consumo de biomasas. Entre los sectores, se producirá una creciente participación de la industria, el transporte y los servicios, en detrimento del sector residencial, en tanto que éste registrará una mayor presencia de los hogares urbanos, variando su consumo según la secuencia que representa la gráfica 3. La demanda se desplaza hacia arriba y hacia la derecha, es decir, hacia combinaciones de consumo con más derivados de petróleo (y de mejor calidad) y más energía eléctrica.

En esas condiciones, la continuidad del rápido crecimiento de la economía y la modificación de las pautas de consumo, inducidas por el aumento del grado de urbanización y el mayor ingreso de los hogares, seguirán elevando esa dependencia exterior ${ }^{9}$ en una proporción que sólo en parte podrán mediatizar la evolución de la producción interna y la reestructura organizativa del sector energético, como se analiza a continuación.

\section{Potencial de la producción interna}

El país cuenta con grandes reservas de carbón, pues, según la estimación de British Petroleum (BP) (2006), la relación reservas-producción actual equivale a 217 años.

9 La IEA (2004) considera que la producción de petróleo crudo se mantendrá bastante estable durante las dos próximas décadas, en torno a los 35-40 millones de toneladas, mientras que la demanda de petróleo se hará incrementado 80\% entre 2002 y 2020 (hasta 224 millones de toneladas), de modo que las importaciones se multiplicarán por 2.2 y la relación entre las importaciones y la demanda superará $85 \%$, o tal vez un porcentaje mayor, que seguirá subiendo en la siguiente década hasta situarse en 90-95\%. También irá en aumento la importación de gas natural, iniciada en 2005, conforme ese recurso vaya acrecentando su presencia en la generación de electricidad y en el consumo de la industria y los hogares. Y proseguirá, probablemente, la importación de ciertas cantidades de carbón, sobre todo coque destinado a la siderurgia. 
Por tanto, su disponibilidad es más amplia, aunque no está exenta de algunos inconvenientes, relacionados con la escasez de coque, la baja productividad de la actividad extractiva, ${ }^{10}$ la carencia de técnicas de lavado para reducir las impurezas, las malas condiciones del transporte ferroviario — por donde se traslada $60 \%$ de la producción, a grandes distancias - y el impacto ambiental que provocan las crecientes emisiones de $\mathrm{CO}_{2}$ y otros gases de efecto invernadero (ECEP, 2005; Ishida 2006). Se trata de un recurso abundante cuya utilización ventajosa depende de que se realicen importantes inversiones para modernizar la actividad extractiva, mejorar el transporte ferroviario y reducir el impacto ambiental.

La situación es diferente en el petróleo y el gas natural. Las estimaciones de BP, Oil and Gas Journal y otras instituciones especializadas coinciden en señalar que las reservas de petróleo son reducidas y apenas se han descubierto nuevas en las últimas décadas (Ishida, 2006; Osumi, 2006). En esas condiciones, la producción de petróleo crudo sólo ha crecido $0.7 \%$ anual entre 1991 y 2004, alcanzando un nivel de 38 millones de toneladas, dos tercios de los cuales se extrae offshore (Ishida, 2006).

Las posibilidades del gas natural son algo mayores. Las reservas probadas equivalen a 36 años del (exiguo) nivel de producción actual, aunque, a diferencia del petróleo, se siguen descubriendo nuevos yacimientos, de modo que es previsible que la producción crezca a buen ritmo en los próximos años. A partir de un nivel muy bajo, la producción ha crecido con celeridad desde 1990, pero su cifra actual, 30 mil millones de $\mathrm{m}^{3}$, sigue siendo modesta. Por ello, la pretensión de incrementar de forma notable la participación del gas en la generación de electricidad, en la industria y en el consumo doméstico, impondrá la necesidad de realizar importaciones, tanto mayores cuanto más se eleven esas demandas internas.

La mayor utilización de recursos no fósiles es la otra opción que puede permitir una cierta sustitución del carbón como recurso mayoritario en la producción de electricidad. La capacidad instalada en plantas hidroeléctricas es débil (32 mil megavatios) y se ha incrementado muy poco durante las últimas décadas, por lo que la producción crece lentamente (1.4\% anual entre 1991 y 2004). Su potencial bruto es bastante amplio, pero se reduce de manera considerable cuando se toman en cuenta bajo criterios de rentabilidad, viabilidad tecnológica, impacto ambiental y gestión de

10 La productividad (media tonelada por empleado en las minas subterráneas y dos toneladas/ empleado a cielo abierto) es casi cinco veces menor que en Estados Unidos, debido al aumento de la profundidad de las extracciones, pero sobre todo al atraso tecnológico de los equipos e instalaciones y a las anticuadas formas de gestión de la minería. 
los terrenos de zonas habitadas. Por esa razón, es previsible que la producción hidroeléctrica aumente de forma paulatina, limitada por las restricciones que imponen las exigencias financieras y los obstáculos mencionados.

La energía nuclear es el centro de un programa muy ambicioso para elevar con rapidez la modesta capacidad actual (3,310 megavatios) de que disponen las 14 plantas que se hallan en funcionamiento. Los planes del gobierno pretenden alcanzar 40 mil megavatios a lo largo de las tres próximas décadas, para que las plantas nucleares aporten la cuarta parte de la producción eléctrica, frente a 3\% actual (Madan, 2006). No obstante, la viabilidad de ese programa nuclear ofrece serias dudas ya que se trata de proyectos que exigen una ingente inversión y una gran capacidad de gestión. Además, las reservas de uranio sólo podrán abastecer una capacidad nuclear de unos 10 mil megavatios, de modo que el resto tendría que importarse, tratándose de un producto de gran sensibilidad geoestratégica. Por otra parte, India es uno de los países no adheridos al Tratado de No Proliferación Nuclear, por lo que su programa nuclear, aunque sea civil, está sujeto a negociaciones político-militares en la escena internacional, en particular con Estados Unidos, que hasta hace pocos años se opuso a la realización de esos proyectos indios.

Las fuentes alternas renovables tienen una presencia testimonial en la oferta energética, aunque algunas disponen de un potencial evidente, que podrá hacerse realidad, de forma paulatina, sobre todo la contribución eólica (Madan, 2006). También la energía solar puede desarrollarse, mientras las biomasas pueden utilizarse para la cogeneración de electricidad y calor, así como para la obtención de biodiesel y etanol.

Así pues, una estimación razonable aconseja prever que en los próximos años se producirá un aumento de la aportación de los combustibles no fósiles en la generación de electricidad, si bien la mayor parte de ésta seguirá procediendo de las centrales térmicas que utilizan carbón, con un incremento notable, pero con una cuota todavía menor, del gas natural. La magnitud que alcance el aumento de la producción eléctrica y la amplitud con la que se diversifique el mix de combustibles dependerá de cómo se afronte el cúmulo de dificultades que se han señalado; entre las que figura en primer plano la capacidad para financiar las grandes inversiones que requiere la mayor producción de carbón y gas natural, y la construcción de nuevas instalaciones hidráulicas, nucleares y eólicas. ${ }^{11}$

11 Según las previsiones de inversión de la IEA (2003), 84\% de los desembolsos que realizarán en el sector energético tendrían que ir a la energía eléctrica, $40 \%$ a generación y el resto a transmisión y distribución.

Vol. 39, núm. 154, julio-septiembre / 2008

Desarrollo 
Reestructuración de las actividades energéticas: eficiencia e inversiones

Esos grandes desembolsos en inversiones tendrán que surgir del incremento de la tasa de ahorro interno que consiga la economía en el curso de su rápido crecimiento, junto con las entradas de capital de los inversionistas extranjeros. Pero, a su vez, este segundo componente dependerá de la marcha del primero y de cómo se lleve a cabo la reestructuración de las actividades energéticas. La estatalización del sector energético comenzó a mitigarse a raíz de las reformas económicas de los noventa, tratando de lograr un funcionamiento más eficiente y de incorporar al capital privado en muchas actividades que hasta entonces le estaban vetadas. En ese proceso, se han producido modificaciones en varios ámbitos, pero muchos otros se mantienen bajo las mismas condiciones organizativas.

El gobierno central, refrendado un sistema parlamentario democrático, pretende seguir con los principales resortes de decisión para garantizar la realización de los objetivos económicos y energéticos que establece por medio de los planes que aprueba para cada lustro. ${ }^{12}$ Pero al mismo tiempo los gobiernos de los estados pretenden asumir esa dirección en su ámbito territorial. La situación se repite a escala de los ministerios ramales, aflorando tensiones entre los organismos centrales y estatales. Bajo otras coordenadas, sucede lo mismo con las agencias reguladoras cuya gestión es independiente del gobierno, pero a veces debe enfrentarse a éste o a los gobiernos estatales cuando adoptan decisiones que mediatizan o impiden su función reguladora (Madan, 2006; ECEP, 2005).

En el plano empresarial, la extracción de petróleo se halla controlada en $87 \%$ por dos empresas públicas (ONGC y OIL) y el restante 13\% corresponde a compañías privadas, con una pequeña presencia de firmas extranjeras (TERI, 2005; Madan, 2006; Ishida, 2006). En el gas natural, esas dos empresas públicas controlan $65 \%$ de la producción, de modo que las compañías privadas extraen ya 35\% de la producción, con una presencia reducida pero significativa de empresas extranjeras (Madan, 2006). En

12 La Planning Commission es el organismo responsable de gestionar el cumplimiento de los objetivos fijados por los plantes quinquenales. La ejecución específica de los proyectos de cada fuente energética corresponde a los respectivos Ministerios de Petróleo y Gas, Carbón, Electricidad, Fuentes No-Convencionales y Energía Nuclear. En un plano distinto figuran las agencias reguladoras que se encargan de fijar las condiciones bajo las que deben actuar las empresas de cada rama energética, con el propósito de hacer compatibles varios objetivos: competencia entre empresas, acceso a las redes de distribución, precios asequibles para los consumidores y cobertura del extenso territorio nacional.

\section{Désarrorollo}

Vol. 39, núm. 154, julio-septiembre / 2008 
la producción de derivados, una empresa estatal (IOC) dispone de $40 \%$ de la producción y casi la mitad de las ventas; mientras que otras ocho empresas públicas cubren alrededor de la tercera parte de la producción y del mercado. Por tanto, el sector privado, liderado por Reliance Petroleum, dispone de la cuarta parte de la producción y la quinta del mercado, siguiendo un proceso cada vez más expansivo al que se van incorporando algunas empresas extranjeras. En el transporte de esos combustibles, la propiedad de los oleoductos recae en una empresa pública (IOC) y la transmisión y distribución de gas natural está monopolizada por otra (GAIL), en tanto que siete compañías públicas controlan la red de productos refinados, junto con tres empresas privadas que comercializan el restante $15 \%$.

En el carbón, la producción y gran parte de la comercialización recae en un oligopolio de compañías estatales, si bien una disposición reciente autoriza la entrada de empresas privadas en la venta al sector residencial. En el sector eléctrico, el gobierno central dispone de $30 \%$ de la producción, correspondiendo otro $60 \%$ a los gobiernos estatales, de modo que la actividad privada controla alrededor de la décima parte (Hansen y Bower, 2003; Ghosh, 2002). La red de transmisión y distribución pertenece a compañías estatales, por lo que tanto las tres empresas del gobierno central que controlan su producción térmica (NTPCL), hidráulica (NHPCL) y nuclear (NNPCIL), como las empresas privadas deben vender su producción a esas compañías de los gobiernos estatales que actúan como mayoristas únicos en sus respectivos territorios. Las firmas privadas sí participan, de forma aún minoritaria, en la fase de comercialización a los consumidores finales.

Por tanto, la mayor parte de la oferta energética sigue bajo control estatal. El sector privado es minoritario, aunque va ganando presencia en la producción y venta de refino y de electricidad, así como en la extracción de gas y petróleo crudo. Se trata de cambios de cierto alcance respecto de la situación anterior a las reformas, pero resultan poco relevantes desde la pretensión de que el mercado sea el mecanismo que prevalezca en las actividades energéticas.

Se crea así un nudo de tensiones a varias bandas. De un lado, entre los poderes públicos (gobierno central, gobiernos estatales y empresas públicas) y el sector privado, representado por las empresas ya existentes, por otros capitales nacionales que aspiran a entrar en el negocio energético y por las empresas transnacionales y los organismos internacionales (FMI y BM) partidarios de la máxima liberalización. Aquéllos se arrogan la representación de los intereses nacionales y éstos reclaman las prerrogativas del mercado. De otro lado, surgen tensiones entre las empresas y los organismos reguladores, cuyas disposiciones son cuestionadas por las compañías

Vol. 39, núm. 154, julio-septiembre / 2008

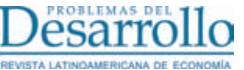


privadas o bien públicas, y otras veces ambas unen sus voces contra las disposiciones reguladoras (Madan, 2006). Y, de otro lado, las tensiones crecen dentro del ámbito público, porque las grandes empresas aspiran a mayores márgenes de autonomía en la toma de decisiones para hacer un uso pleno de sus posiciones mayoritarias o monopolistas en sus respectivas ramas energéticas (Srivastava y Mathur, 2007).

Se trata de situaciones conflictivas que cobran más o menos intensidad según los momentos, pero que emplazan a una pugna estratégica de largo plazo que tendrá que ir decantándose en el curso de los próximos años. Por una parte, los poderes públicos desean seguir conduciendo la transformación de la economía y, dentro de ella, los cambios que requiere el sistema energético. Disponen para ello de los mecanismos de planificación, de la propiedad mayoritaria de las empresas y del control administrativo de la gestión energética y se muestran dispuestos a ampliar paulatinamente —bajo su control— los márgenes de participación del sector privado en las actividades energéticas. Por otra parte, el gran volumen de inversiones que requieren sus planes energéticos hace que los dirigentes indios recaben cada vez con más insistencia la participación del capital privado nacional y de capitales extranjeros en esas inversiones, pero éstos reclaman mayores márgenes de liberalización para participar.

Con esa línea, ya está permitida la creación de empresas con capital íntegramente extranjero, sin necesidad de asociarse con empresas públicas o privadas nacionales. Igualmente, en 1997 se puso en marcha la New Exploration Licensing Policy (NELP), por la que se conceden licencias para explorar y extraer petróleo y gas, con abundantes concesiones para las compañías extranjeras. Sin embargo, ésas y algunas otras reformas no parecen ser condiciones suficientes que atraigan volúmenes importantes de capital foráneo, ya que la "cartera de reivindicaciones" de los capitales extranjeros es bastante más radical que las pretensiones del gobierno. Sus objetivos apuntan a la privatización de las grandes empresas estatales, a la ruptura de las actuales estructuras verticales sobre el conjunto de las fases del ciclo de cada recurso energético y a la reducción de las regulaciones públicas sobre el funcionamiento de los mercados.

Por tanto, las autoridades se enfrentan así ante un formidable dilema entre lo que quieren (conducir la transición energética) y lo que necesitan (inversiones privadas), lo que previsiblemente dará lugar a una liberalización cada vez mayor e incluso la privatización de empresas públicas, registrándose una aportación creciente de capitales privados, en buena parte extranjeros. La incertidumbre tiene que ver con el ritmo que adopte esa liberalización y la envergadura que alcancen las entradas de capital, si bien el escenario más verosímil es que no habrá alteraciones abruptas, sino un proceso paulatino que se prolongará durante bastante tiempo, dilatando la travesía que 
supone la transición energética hacia un sistema más acorde con los requerimientos del desarrollo económico.

\section{Conclusiones}

Los principales rasgos del sistema energético de India siguen siendo los propios de un país escasamente desarrollado, tanto por la reducida dimensión de la demanda de energía como por la escasa presencia de los sectores productivos (se concentra en el sector residencial) y la poca diversificación de los recursos consumidos (concentrados en madera y desechos). Las novedades afectan sobre todo al aumento del consumo final de derivados de petróleo y de electricidad, al desajuste de la oferta interna-demanda de petróleo y al fuerte aumento de las importaciones de petróleo crudo.

La dinámica económica y social del país explica las condiciones energéticas, tanto en los factores que garantizan la continuidad de los rasgos del sistema tradicional como en los factores que determinan los cambios incipientes. La nueva diversificación hacia una mayor presencia de los derivados de petróleo y de energía eléctrica está determinada por el dinamismo que adquieren las industrias que trabajan en condiciones de outsourcing para compañías internacionales y, sobre todo, por el incremento del ingreso y el leve aumento de la población que vive en las ciudades. Los hogares urbanos y una parte de los hogares rurales con mayores niveles de renta elevan su consumo de energía y modifican la estructura de sus necesidades, dando lugar a que su demanda se desplace hacia el keroseno, diesel, gases de petróleo y energía eléctrica.

La capacidad de producción interna permite atender al aumento de la demanda de electricidad, aunque de forma insuficiente, mediante el incremento de la extracción de carbón y, en menor medida, gas natural y combustibles no fósiles. Sin embargo, no ocurre lo mismo con la demanda de derivados de petróleo, pues la producción del energético crudo es reducida, de modo que una parte creciente de la capacidad de refino se efectúa con crudos importados. Por esa razón, las compras de éstos en el exterior se acercan a $80 \%$ de la demanda interna de petróleo. A la vez, esas refinerías disponen de una estructura de productos refinados que se orienta al mercado interno, aunque con un cierto desajuste, ya que en la actualidad se exporta alrededor de la décima parte de la producción y se importan cantidades menores de algunos productos deficitarios.

Los objetivos económicos del gobierno y las previsiones de los organismos internacionales señalan que India mantendrá un alto ritmo de crecimiento durante las próximas décadas. Con ese horizonte, la dinámica de desarrollo dependerá de la ca- 
pacidad que muestre la economía para incorporar una especialización productiva más moderna y para fortalecer sus infraestructuras. En la medida en que avance por ese camino, el incremento de la renta unido a la expansión de nuevas ramas productivas (finales e intermedias), el fortalecimiento del sector de transporte y el mayor grado de urbanización exigirán un mayor consumo energético y una diversificación mayor de la demanda, superando los rasgos característicos del sistema energético tradicional. Aumentará bastante más la presencia de los derivados, la electricidad y el gas natural en la demanda final, al igual que la del gas y los combustibles no fósiles en la generación de electricidad, aunque el carbón seguirá siendo el recurso dominante en las plantas térmicas.

Para adentrarse en ese proceso de transformación energética, India tendrá que realizar un gran esfuerzo productivo. Para ello cuenta con recursos abundantes de carbón destinados en su mayoría a esas plantas térmicas, a la vez que están en marcha planes de expansión para la energía nuclear, las energías renovables y el gas natural. Todo ello permitiría afrontar el reto que supondrá el aumento de la demanda de electricidad, aunque para esto se necesita un volumen de inversiones que requiere la participación del sector privado, nacional y extranjero. Sin embargo, estos capitales privados condicionan su participación en las inversiones a que el gobierno promueva una liberalización bastante más profunda, se modifiquen las estructuras verticales y se favorezca la privatización de las empresas estatales que dominan de forma abrumadora la producción y el transporte de todas las ramas energéticas.

Las incertidumbres que arroja ese dilema entre los proyectos expansivos del gobierno y la necesidad de realizar mayores concesiones a los inversionistas privados se irán dilucidando mediante tensiones más o menos intensas que, previsiblemente, perdurarán durante un largo periodo. Sin embargo, no admite dudas el otro hecho fundamental que presidirá la transición energética: el aumento de las importaciones de petróleo. La mayor parte del incremento de la demanda de derivados tendrá que cubrirse con compras en los mercados exteriores. Es razonable prever que la dependencia de exterior se acerque a $90 \%$ de la demanda en el curso de la próxima década, previendo también un aumento de las importaciones de gas natural y de uranio para las plantas nucleares.

\section{DeSTarrollo}

Vol. 39, núm. 154, julio-septiembre / 2008 


\section{Bibliografía}

Asian Development Bank, Key Indicators of Developing Asian and Pacific Countries, 2007, www.adb.org.

Bajpai N., India in the Era of Economics Reforms, Delhi, Oxford University Press, 1999.

British Petroleum, Statistical Review of World Energy, 2006, www.bp.com/centres/energy.

Central Statistical Organization, Energy Statistics 2000-2005, Delhi, Ministry Statistics and Programme Implementation, 2006.

Chai, J. y K. Roy, Economic Reform in China and India, Cheltenham, Edward Elgar, 2006.

Chauvin, S. y F. Lemoine, "L'économie indienne: changements structurels et perspectives à long-terme", Document du travail 04, CEPII, abril, 2005.

Expert Committee on Energy Policy (ECEP), Draft Report of the Expert Committee on Integred Energy Policy, Nueva Delhi, Planning Commission, 2005.

Ghosh, S., "Electricity consumption and economic growth in India", Energy Policy, 30 (2), 2002, pp. 125-29.

Hansen, Ch. y J. Bower, "Political Economy of Electricity Reform. A case Study in Gujarat, India", Oxford Institute for Energy Studies, EL 03, septiembre, 2003.

International Energy Agency (IEA), India - A Growing International Oil and Gas Player, París, IEA, OCDE, 2000.

World Energy Investment Outlook, Asia-Pacific Energy Investment Challenger, París, IEA, OCDE, 2003.

, Energy Balances of non-OECD Countries, 1971-2004, París, IEA Statistics CD, OCDE, 2006a.

Oil Information, IEA Statistics CD, París, OCDE, 2006b.

, Natural Gas Information, IEA Statistics CD, París, OCDE, 2006c.

Ishida, H., Energy Situation and Policy in India, Institute of Energy Economics, Tokyo, 2006, http://eneken.ieej.org/en.

, Energy Strategies in China and India and Major Countries 'Views, Institute of Energy Economics, Tokyo, marzo, 2007, http://eneken.ieej.org/en.
Judson, R., R. Schmalensee y M. Stoker, "Economic Development and the Structure of the Demand for Commercial Energy", The Energy Journal, 20 (2), 1999, pp. 29-57.

Koizumi, K. et al., Coal supply and Demand Trends in India, Institute of Energy Economics, Tokyo, octubre, 2006, http:// eneken.ieej.org/en.

Madan, T., India, The Bookings Foreign Policy Studies, Energy Security Series, Washington, noviembre, 2006.

Osumi, India and China'Energy Strategies in Middle Eastern Oil-Producing Nations, Tokyo, The Institute of Energy Economics, diciembre, 2006, http://eneken.ieej.org/en.

Perlitz, U., "Steel market in India: Companies set for expansion", Deutsche Bank Research, India Special, Current Issues, 27 de septiembre, 2007.

Planning Commission Government of India, PCGI, India Vision 2020, Report of the Committee on India Vision 2020, Delhi, PCGI, 2002.

Schipper, L. y M. Grubb, "On the rebound? Feedback between energy intensities and energy uses in IEA countries", Energy Policy, 28, (6-7), 2000, pp. 367-88.

Srinivasan, T., Reintegrating India with the World Economy, Washington, Institute for International Economy, 2003.

Srivastava, L. y R. Mathur, "India's Energy Security", Briefing Paper, 14, FES, New Delhi Friedrich Eberg Stiftung, septiembre, 2007.

The Economist Intelligence Unit (EIU), India, Country Profile, Londres, EIU, 2007.

The Energy and Ressources Institute (TERI), Energy Directory \& Yearbook 2004/05, Basic Statstics on Indian Petroleum \& Natural Gas, Delhi, Minstry of Petroleum \& Natural Gas, 2005.

Virmani, R., "India's Economic Growth History: Fluctuations, Trends, Break Points and Phases", Occasional Paper, Indian Council for research on International Economic Relations, enero, 2005, www. icrier.org.

Yi-chong, X., Electricity Reform in China, India and Russia, Washington, World Bank, Washington, 2004. 


\section{ECONOMIAUnam}

- Politica energética de Estados Unidos

- Tarifas en el servicio de suministro de turbosina

- Propuesta de crecimiento para México

- ¿A quién benefician las remesas?

Universidad Nacional

Autónoma de México 